\title{
APPROXIMATION BY SEMI-FREDHOLM OPERATORS
}

\author{
FERNANDO GALAZ-FONTES
}

(Communicated by Palle E. T. Jorgensen)

\begin{abstract}
We present a simple approach for calculating some known distances from a bounded operator defined on a separable Hilbert space to certain sets related to semi-Fredholm operators.
\end{abstract}

\section{INTRODUCTION}

There are several semigroups which appear naturally in connection with the Banach algebra $B(X)$ of all (bounded linear) operators acting from a Banach space $X$ into itself. We have the injective operators

$$
\operatorname{Inj}(X)=\{T \in B(X): T \text { is one-to-one, and } R(T) \text { is closed }\},
$$

the surjective operators

$$
\operatorname{Sur}(X)=\{T \in B(X): R(T)=X\},
$$

the upper semi-Fredholm operators

$$
\Phi_{+}(X)=\{T \in B(X): \operatorname{dim} N(T)<\infty \text { and } R(T) \text { is closed }\},
$$

and the lower semi-Fredholm operators

$$
\Phi_{-}(X)=\{T \in B(X): \operatorname{dim} X / R(T)<\infty\} ;
$$

here $N(T)$ denotes the null space of $T$ and $R(T)$ is its range. It is well known that these semigroups are open [5, Theorem 2.5.6 and 4.2.1], and we clearly have $\operatorname{Inj}(X) \subset \Phi_{+}(X)$ and $\operatorname{Sur}(X) \subset \Phi_{-}(X)$. Although the contentions are generally proper, Lemma 2.1 shows that $\operatorname{Inj}(X) \cup \operatorname{Sur}(X)$ is dense in $\Phi_{+}(X) \cup \Phi_{-}(X)$.

Recall that the index of a (upper or lower) semi-Fredholm operator $T$ is ind $T=n(T)-d(T)$, where $n(T)=\operatorname{dim} N(T)$ and $d(T)=\operatorname{dim} X / R(T)$. Let $Z^{*}$ consist of all the integers together with $-\infty$ and $\infty$. For $m \in Z^{*}$, we define $\Phi_{m}(X)$ as the set of semi-Fredholm operators $T \in B(X)$ such that ind $T=m$.

If $H$ is a complex separable Hilbert space, Bouldin [2-4], Zemánek [8], Apostol et al. [1], Izumino and Kato [6], and $\mathrm{Wu}$ [7] have calculated the distance

Received by the editors July 30, 1992.

1991 Mathematics Subject Classification. Primary 47A53, 47A55.

Key words and phrases. Semi-Fredholm operator, index, injective operator, surjective operator, essential minimum modulus.

This work was supported by CONACYT. 
of $T \in B(H)$ to several subsets related to semi-Fredholm operators on $H$. We develop an alternative approach for obtaining some of their results.

\section{APPROXIMATION BY SEMI-FREDHOLM OPERATORS}

Let us consider

$$
F_{m}(X)= \begin{cases}\Phi_{m}(X) \cap \operatorname{Sur}(X), & m \geq 0 \\ \Phi_{m}(X) \cap \operatorname{Inj}(X), & m \leq 0 .\end{cases}
$$

The following result is probably well known, but we have not found it stated explicitly. We present it here for the sake of completeness.

Lemma 2.1. $F_{m}(X)$ is dense in $\Phi_{m}(X), m \in Z^{*}$.

Proof. First we will assume that $m \leq 0$. Take $T \in \Phi_{m}(X)$. Since $m \leq 0$, note that $n=n(T)<\infty$. Thus we can express $X=N(T) \oplus V$, for some closed subspace $V \subset X$. Next, by using $d(T) \geq n$, we can find a vector space $W \subset X$ such that $\operatorname{dim} W=n$ and $W \cap R(T)=0$. Take $S$ to be any linear isomorphism from $N(T)$ onto $W$. Let us define $T_{k}: X \rightarrow X$ by

$$
T_{k}(u+v)=\frac{1}{k} S u+T v, \quad u \in N(T), v \in V .
$$

Since the projections onto $N(T)$ and $V$ are continuous, as well as $S$, it follows that $T_{k} \in B(X)$. It is clear that $T_{k} \in \operatorname{Inj}(X) \cap \Phi_{m}(X)$. Noticing that $T_{k} \rightarrow T$, we obtain the desired conclusion.

Let us now consider $m \geq 0$. Then we have $d=d(T)<\infty$, so we can express $X=N \oplus V$, where $N \subset N(T)$ and $\operatorname{dim} N=d$. The rest of the proof follows along similar lines as above, and we will omit it.

Remark 2.1. The proof of Lemma 2.1 shows that if $T \in \Phi_{m}(X)$, then there is a compact operator $K \in B(X)$ such that $T+K \in F_{m}(X)$.

It will now be convenient to introduce some notation. Hereafter $H$ is always a fixed complex separable Hilbert space. If $A \subset H$, then $A^{\mathrm{c}} \equiv H \backslash A$; for $T \in B(H), T^{*}$ is its adjoint operator and $|T|$ is the square root of $T^{*} T$. The group of invertible operators on $H$ will be indicated by $G(H)$; moreover, $\Phi_{+} \equiv \Phi_{+}(H), \Phi_{-} \equiv \Phi_{-}(H), \Phi_{m} \equiv \Phi_{m}(H)$.

Let us denote the semigroup of Fredholm operators on $H$ by $\Phi$; notice that $\Phi=\Phi_{+} \cap \Phi_{-}$. The essential spectrum of $T \in B(H)$ is then given by $\sigma_{e}(T)=\{\lambda: T-\lambda I \notin \Phi\}$, and the essential minimum modulus is

$$
m_{e}(T)=\inf \left\{\lambda: \lambda \in \sigma_{e}(|T|)\right\} .
$$

Our approach for calculating the distance $\operatorname{dist}(T, A)$ of $T \in B(H)$ to certain subsets $A$ of $B(H)$ relies on the set

$$
N(H)=\left\{T \in B(H): m_{e}(T)=m_{e}\left(T^{*}\right)=0\right\},
$$

together with the following result (part(i) is due to Bouldin [2, Theorem 3], and part (ii) is due to Zemánek [8]).

Theorem 2.1. (i) If $T \in N(H)$, then $\operatorname{dist}(T, G(H))=0$.

(ii) $m_{e}(T)=\operatorname{dist}\left(T, \Phi_{+}^{\mathrm{c}}\right), m_{e}\left(T^{*}\right)=\operatorname{dist}\left(T, \Phi_{-}^{\mathrm{c}}\right)$.

Remark 2.2. $N(H)$ is a closed subgroup of $B(H)$. 
Proof. Let $T \in B(H)$. The following properties are well known [2]:

$$
\begin{array}{cll}
m_{e}(T)>0 & \text { if and only if } & T \in \Phi_{+}, \\
T \in \Phi_{-} & \text {if and only if } & T^{*} \in \Phi_{+} .
\end{array}
$$

This implies that $N(H)=\left(\Phi_{+} \cup \Phi_{-}\right)^{\mathrm{c}}$, so $N(H)$ is clearly closed.

Assume, $S, T \in N(H)$. Since

$$
T S \in \Phi_{-} \quad \text { implies } T \in \Phi_{-}
$$

and $T S \in \Phi_{+}$implies $S \in \Phi_{+}$[5, Corollary 1.3.3], we have $T S \in N(H)$.

In our next result bdy $A$ denotes the boundary of $A \subset B(H)$.

Theorem 2.2. For each $m \in Z^{*}$, we have $N(H)=\operatorname{bdy} \Phi_{m}$.

Proof. By the continuity of the index on $\Phi_{+} \cup \Phi_{-}$, it follows that bdy $\Phi_{m} \subset$ $N(H)$.

Next, let us assume $m \in Z \cup\{-\infty\}$. Fix $R \in \Phi_{m} \subset \Phi_{+}$. Take $P$ and $Q$ to be the orthogonal projections onto the null space and the range of $R$, respectively, and let $U$ be the inverse operator of $T:(I-P) H \rightarrow R(H)$. Then $U Q R=I-P$, so, with $S=U Q$, we have $S \in B(H)$ and

$$
S R=I+K,
$$

where $K \in B(H)$ is a compact operator. Take $T \in N(H)$, and note that $T \notin \Phi_{m}$. By (1), $T S \notin \Phi_{-}$. Suppose that $T S \in \Phi_{+}$. Then, using (2), we would have $T \in \Phi_{+}$, which is contradictory.

The above shows that $T S \in N(H)$. Thus, by (i) of Theorem 2.1, there is a sequence $\left\{L_{k}\right\} \subset G(H)$ such that $L_{k} \rightarrow T S$. Hence, applying (2), we have $L_{k} R \rightarrow T+T K$. Since ind $\left(L_{k} R-T K\right)=m$, it follows that $T \in \operatorname{bdy} \Phi_{m}$. This shows that $N(H)=\operatorname{bdy} \Phi_{m}$.

Finally let us treat the case $m=\infty$. Take $T \in N(H)$. Then $T^{*} \in N(H)$, so, applying what we have just proved, there is a sequence $\left\{S_{k}\right\} \subset \Phi_{-m}$ such that $S_{k} \rightarrow T^{*}$. Hence $S_{k}^{*} \rightarrow T$, and the conclusion follows.

Corollary 2.1. Let $m \in Z^{*}$. If $T \notin \Phi_{m}$, then

$$
\operatorname{dist}\left(T, \Phi_{m}\right)=\max \left\{m_{e}(T), m_{e}\left(T^{*}\right)\right\} .
$$

Proof. First, we notice that $m_{e}(T)=m_{e}\left(T^{*}\right)$ when both of these quantities are positive [2, Theorem 2]. Thus

$$
\begin{array}{ll}
\max \left\{m_{e}(T), m_{e}\left(T^{*}\right)\right\}=m_{e}(T) & \text { if } T \in \Phi_{+}, \\
\max \left\{m_{e}(T), m_{e}\left(T^{*}\right)\right\}=m_{e}\left(T^{*}\right) & \text { if } T \in \Phi_{-} .
\end{array}
$$

Take $d \equiv \operatorname{dist}\left(T, \Phi_{m}\right)$. We will consider several cases. If $T \notin \Phi_{+} \cup \Phi_{-}$, the conclusion follows readily from Theorem 2.2 .

Now assume $T \in \Phi_{+}$, and let $0<\rho<m_{e}(T)$. By Theorem 2.1(ii) the closed ball $V$ consisting of those $R \in B(H)$ such that $\|R-T\|<\rho$ is contained in $\Phi_{+}$. Since $V$ is connected, by the continuity of the index, ind $R=\operatorname{ind} T$ when $R \in V$. This implies

$$
m_{e}(T) \leq d .
$$

Let $\varepsilon>0$. Again applying Theorem 2.1, we can obtain $S \notin \Phi_{+}$satisfying $\|T-S\| \leq m_{e}(T)+\varepsilon$. Now note that, because of the continuity of the index on $\boldsymbol{\Phi}_{+} \cup \boldsymbol{\Phi}_{-}$, the curve $\alpha(t)=(1-t) T+t S, 0 \leq t \leq 1$, cannot be contained 
in $\Phi_{+} \cup \Phi_{-}$. Thus we can find $0 \leq t \leq 1$ such that $\alpha(t) \in N(H)$. Since $\|T-\alpha(t)\| \leq m_{e}(T)+\varepsilon$, we have $d \leq m_{e}(T)+\varepsilon$. Letting $\varepsilon \rightarrow 0$ and using (3), the conclusion follows.

Finally, the case $T \in \Phi_{-}$can be proved analogously.

Remark 2.3. Corollary 2.1 was established independently by Apostol et al. [1, Theorem 12.2] and Izumino and Kato [6, Theorem 4.1]; it is also discussed by Bouldin [3].

Corollary 2.2. If $n \leq 0$, then

$$
\operatorname{dist}\left(T, F_{n}\right)= \begin{cases}\max \left\{m_{e}(T), m_{e}\left(T^{*}\right)\right\} & \text { if } \operatorname{ind} T \neq n, \\ 0 & \text { otherwise. }\end{cases}
$$

Proof. It follows from Lemma 2.1 that $\operatorname{dist}\left(T, F_{n}(H)\right)=\operatorname{dist}\left(T, \Phi_{n}\right)$. The conclusion is now obtained by using Corollary 2.1 .

Remark 2.4. Corollary 2.2 was established by $\mathrm{Wu}$ in [7].

Let us denote by $\nu$ the function assigning $n(T)$ to each $T \in B(H)$.

Corollary 2.3. The function $\nu$ is continuous at $T \in B(H)$ if and only if $T \in$ $\Phi_{+} \cup \Phi_{-}$and $n(T)=0$ or $d(T)=0$.

Proof. Let $A \equiv\left\{T \in \Phi_{+} \cup \Phi_{-}: n(T)=0\right.$, or $\left.d(T)=0\right\}$. If $T \notin A$, it follows from Lemma 2.1 and Theorem 2.2 that $\nu$ is discontinuous at $T$. Now assume $T \in A$. Take $\rho>0$ such that $\|T-S\| \leq \rho$ implies $S \in\left(\Phi_{+} \cup \Phi_{-}\right), n(S) \leq$ $n(T)$, and $d(T) \leq d(S)$ [5, Theorem 4.2.1]. If $n(T)=0$ and $\|T-S\| \leq \rho$, we clearly have $n(T)=n(S)$. If $d(T)=0$ and $\|T-S\| \leq \rho$, then $n(T)=n(S)$ because $\operatorname{ind}(T)=\operatorname{ind}(S)$. This proves our assertion.

\section{NOTE ADDED IN PROOF}

After this work was sent for publication, a paper of M. Mbekhta appeared which contained results closely related to ours but using different methods (Sur la structure des composantes connexes semi-Fredholm de $B(H)$, Proc. Amer. Math. Soc. 116 (1992), 521-524).

\section{REFERENCES}

1. C. Apostol, L. A. Failkow, D. A. Herrero and D. Voiculescu, Approximation by Hilbert space operators, Vol. II, Pitman, Boston, MA, 1984.

2. R. Bouldin, The essential minimum modulus, Indiana Univ. Math. J. 30 (1981), 513-517.

3. 139-143.

4. $ـ$, Approximation by operators with fixed nullity, Proc. Amer. Math. Soc. 103 (1988), 141-144.

5. S. R. Caradus, W. E. Pfaffenberger, and B. Yood, Calkin algebras and algebras of operators on Banach spaces, Marcel Dekker, New York, 1974.

6. S. Izumino and Y. Kato, The closure of invertible operator on a Hilbert space, Acta Sci. Math. (Szeged) 49 (1985), 321-327.

7. P. Y. Wu, Approximation by invertible and noninvertible operators, J. Approx. Theory 56 (1989), 267-276.

8. J. Zemánek, Geometric interpretations of the essential minimum modulus in Invariant subspaces and other topics, Oper. Theory, Adv. Appl., vol. 6, Birkhäuser Verlag, Basel, 1982, pp. 225-227.

Centro de Investigación en Matemáticas, A. P. 402, 36 000, Guanajuato, Gto., Mexico E-mail address: cimat@redvax1.dgsca.unam.mx 